\title{
The Design of the Lottery Real-time Monitoring System based on LABVIEW
}

\author{
Meng Li \\ Shanghai University of \\ Engineering Science \\ Songjiang Shanghai \\ 201620, China
}

\author{
Chen Deng \\ Shanghai University of \\ Engineering Science \\ Songjiang Shanghai \\ 201620, China
}

\author{
Yaping Tu \\ Donghua University \\ Songjiang Shanghai \\ 201620, China
}

\begin{abstract}
Analyzing lottery data from the web is often a problem for those who are enthusiastic about lottery. The difficulty of realtime lottery data acquisition and recording is another problem that may set most people down. This paper uses automatic reference control and .NET control provided by LabVIEW to capture the underlying data from web. The obtained information from web pages is then transformed into onedimensional array or two-dimensional array by screening and evaluating it. The result shows that this is an effective way to capture and analysis lottery data. The purpose of this paper is to provide a new method of web data collection and management based on the LabVIEW platform.
\end{abstract}

\section{General Terms}

Data Acquisition

\section{Keywords}

Lottery, Web Information, Alarm, Data Acquisition, LabVIEW.

\section{INTRODUCTION}

China Welfare Lottery was founded in 1987, which is targeted at "uniting all kinds of people who are enthusiastic about social welfare, promoting socialist humanitarian spirit, raising social welfare funds, the establishment of the disabled, the elderly, orphans welfare and helping poor people". This is for the purpose of "helping the old, disabled, save the orphaned, the poor". At present, the vast majority of the punters still rely on manual analysis of historical data. However, too many lottery periods, large amount of data, complexity of calculation, long computing time are plagued by many lottery enthusiasts. Finding a tool that can shorten the calculation time and the analysis data effectively has certain practical significance to lottery fans.

According to the requirements of the punters on the control and data analysis system, the platform needs to have the function of browsing lottery history and real-time data acquisition. With the quick analysis and feedback provided by software after integrating the needed data, customers can bets within the prescribed time successfully and have a higher possibility to win. The emergence of virtual instrument provides us with a high-performance modular hardware, combined with highly efficient and flexible software to complete a variety of applications such as data test, measurement and automation. LabVIEW is a kind of programming language based on G (Graphic) graphical development environment. It has all the standard features of general programming environment, such as data structure, circulation structure and event procession and built-in compiler. However, the difference from other general programming language is that LabVIEW is designed for scientists and engineers. Its various built-in functions, assistants and tools make LabVIEW more than just a programming language, but also the industry and academia summary for development of data acquisition system, instrument control software and analysis tool. Because LabVIEW provides a rich and practical library function and hardware driver library, users can develop their virtual instruments (VIs) in a very short time. And by modifying the code, some VIs can be easily changed, added or removed to meet new requirements.

\section{IDEAS AND PRINCIPLES OF SYSTEM DESIGN}

\subsection{System Structure}

Structures of real-time monitoring Lottery data and control system are shown in Fig.1.The whole system is mainly composed of four parts namely data acquisition module, data analysis module, alarm module and human-machine interface module.

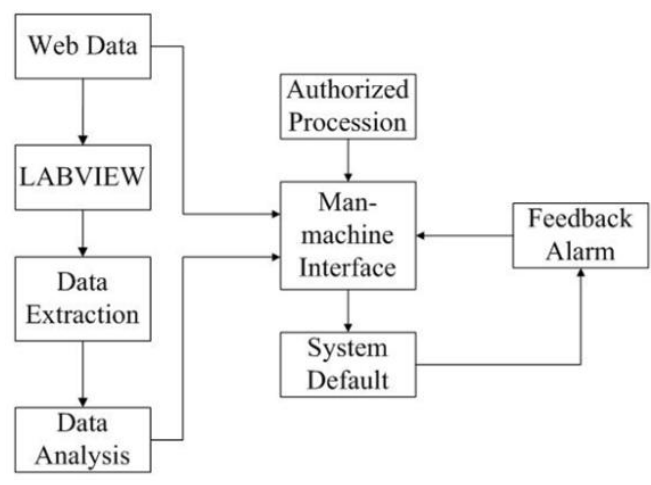

Fig 1: Lottery monitoring system structure

\subsection{System principle}

Firstly, the system provides the IE control, .NET control and automation control by LabVIEW to the control system, and completes preliminary data acquisition and data update. The whole procession continues over time. Finally according to the needs of users, the system uses development tools providing an array control, the cluster control and the chart control as well as the extraction of web information to recombine the information and split analysis objects one by one, and capture the effective information through the manmachine interface displays.

National Instruments provide us with powerful VIs, which make it easy for interacting with the Internet and collecting web data. The validity of the data mainly focuses on the analysis of the category of lottery data, the harness of the regulation so that the seemingly haphazard historical data can be shown clearly. Finishing the lottery periods, analyzing all the data accurately and bringing punters intuitive feelings are 
more important. And the system can also realize the alarm set, automatic alarm remind function, the ideas of the punters bet presented in the form of software.

\section{SYSTEM FUNCTION MODULE DESIGN}

\subsection{Data acquisition module}

First step of obtaining effective information is to extract web data. That is to get data from large amount of web information. This is a crucial step, so the problem is how to use the program to control web pages and how to capture the real-time dynamic information of the page. LabVIEW provides us with effective solutions. One method is to use IE VI and the other method is to use Web Browser .NET VI. Based on the capture effectiveness and dynamic performance of access to information, this paper uses the second method to capture web information. Information acquisition program block diagram is shown in Fig.2.

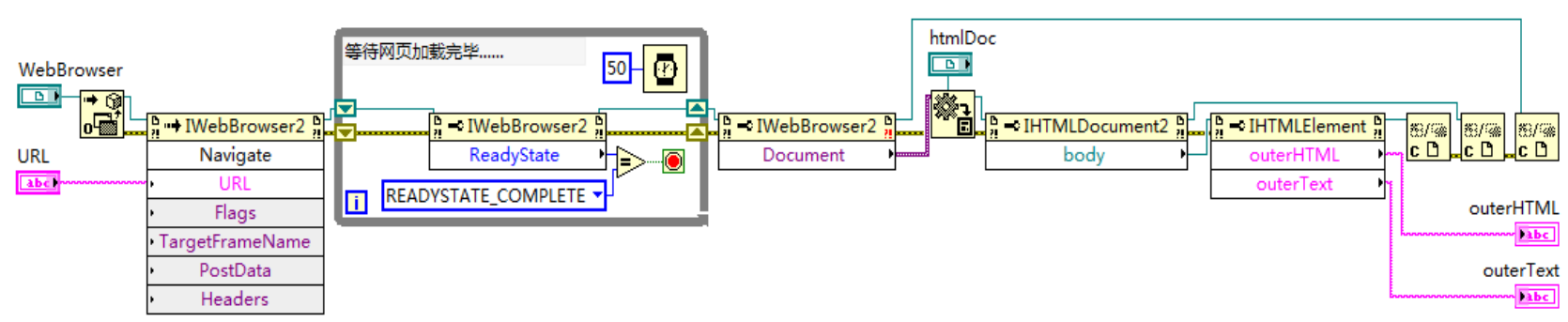

Fig 2: Using the Web Browser VI to extract information from web pages

Data processing is mainly to convert the data obtained from the website for the convenience of analyzing and displaying the results. Originally, the type of collected data is a string of no rules and it can't be directly displayed in human-machine interface. In Fig. 3 for example:

\begin{tabular}{|l|l|}
\hline $659223 \times 014438706209695003396874225246752454427617934$ \\
\hline $659224 \times 042647167530451358512202355303505520281077716$ \\
\hline $659225 \times 017126543274233629665620624611316167254741878$ \\
\hline $659226 \times 020860452004315405435513240775223609722635644$ \\
\hline $659227 \times 014159622453486527544468204736615706604904885$ \\
\hline $659228 \times 016609636732441177415920744550166965390448899$ \\
\hline
\end{tabular}

\section{Fig 3: Collected data string}

The data above shows that six figures at the beginning are the number of the lottery period, but the following three figures are the sum of all the valid numbers. Every two contiguous numbers stands for a single set. According to this rule, this paper chooses the method of string intercept method, and it will capture information displayed in the corresponding text box.

\subsection{Data analysis module}

Data analysis is mainly to complete the calculation of the size, parity and the sum of the numbers. After the collection and processing of the source, data is presented in the form of a one-dimensional array, as it is shown in Fig.3. Using the method of grouping analysis, it will get a dimension of the array elements, in the form of the split rebuilt single dimensional array, each issue can be data size, parity, etc. After restructuring a series of data analysis, and then getting all the historical data, user can get the number what they want to have.

Grouping method is based on the analysis of the characteristics of the object. According to certain signs, analysis of the data object is divided into different parts and types to show its inner link and law. The key to grouping method is to determine the array with the class. In data grouping, the boundary of each group is called group limit. The average value of upper limit and lower limit is called group value.
Contrast analysis refers to two or more data comparison, analysis of their differences, so as to reveal the data represented by the development regularity and the changes of the object.

\subsection{Alarm default module}

Alarm module contains two parts: the default alarm threshold value and the reminder. Users can set alarm threshold on the tool bar. As the web data updates, the software also updates data at the same time. When real-time data reaches alarming expectations, software interface displays audio alarm warning lamp and driver.

\subsection{Human-machine interface module}

Human-machine interface module is the core part of the whole system. It is the bridge between the software and users. Human-machine interface is mainly to complete all historical data retrieval, especially the visual display of historical data analysis results, at the same time it contains historical data period updating and alarm value preset functions.

\section{SYSTEM TEST AND ANALYSIS}

Testing experimental results of system is to ensure that the network is in good condition. Software operation interface as shown in Fig.4. It shows the effective capture of web data, and successfully display the lottery number analysis results on the human-machine interface. At the same time it completes the alarm function according to the requirement excellently. 


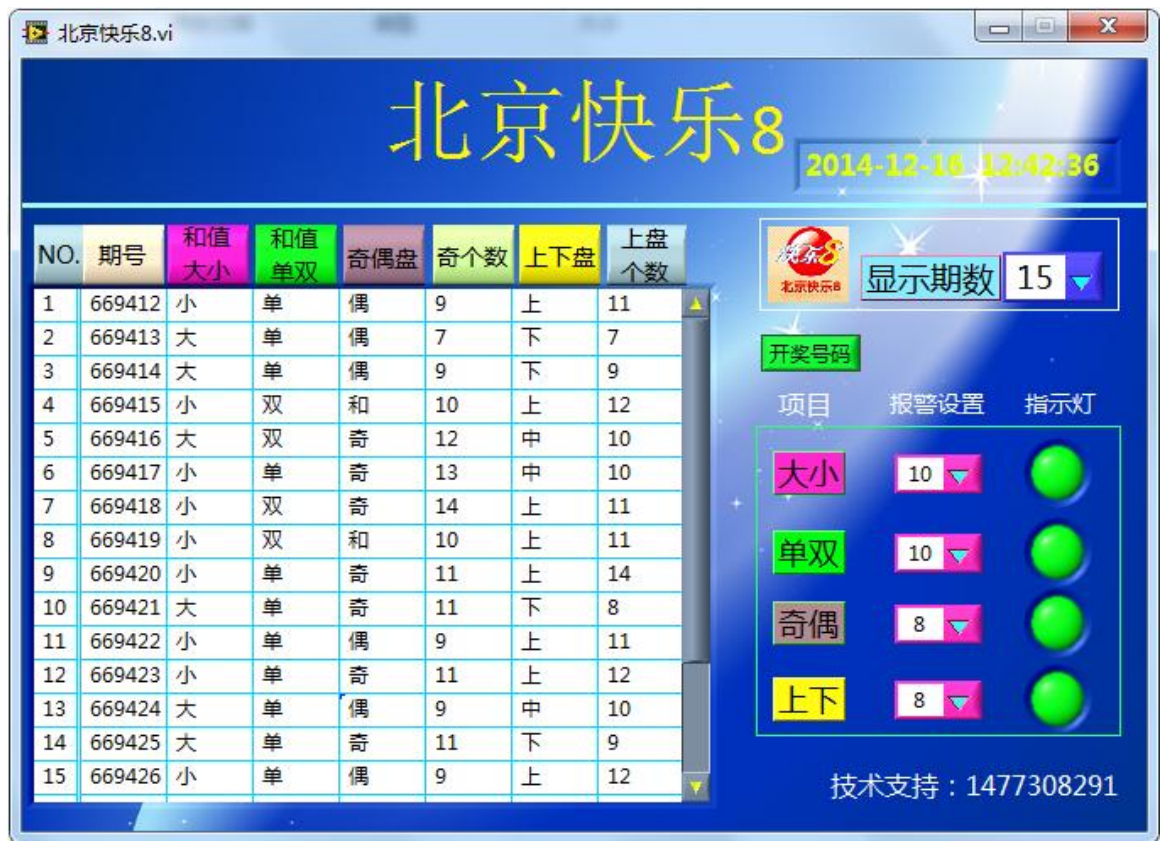

Fig 4: Human-machine interface

\section{COMPARATIVE ANALYSIS}

Fig.5 shows the web information, in serial number, 669426 period for example, the lottery number is $14,67,58,23,07,39,59,08,50,62,21,30,13,76,29,45,16,68,38,40$, among which $67,23,07,39,59,21,13,29,45$ are 9 odd numbers,

\begin{tabular}{|c|c|c|c|}
\hline 669426 & $2014-12-16$ 12:35:00 & (01) & $\begin{array}{l}14,67,58,23,07,39,59,08,50,62 \\
21,30,13,76,29,45,16,68,38,40\end{array}$ \\
\hline 669425 & $2014-12-16$ 12:30:00 & $x$ & $\begin{array}{l}79,23,54,37,80,19,39,31,71,58 \\
14,65,44,02,35,64,76,20,51,41\end{array}$ \\
\hline 669424 & $2014-12-16$ 12:25:00 & & $\begin{array}{l}35,53,26,72,39,45,24,63,58,33 \\
01,42,60,06,57,62,32,38,11,74\end{array}$ \\
\hline 669423 & $2014-12-16$ 12:20:00 & & $\begin{array}{l}29,23,56,45,09,54,35,02,25,52 \\
59,17,40,30,65,22,42,26,73,11\end{array}$ \\
\hline 669422 & 2014-12-16 12:15:00 & & $\begin{array}{l}47,60,23,31,13,71,27,44,22,67 \\
36,41,14,61,48,24,04,40,58,10\end{array}$ \\
\hline 669421 & $2014-12-16$ 12:10:00 & $x 0$ & $\begin{array}{l}49,04,32,63,74,15,53,48,76,20 \\
57,40,77,11,41,25,73,60,10,71\end{array}$ \\
\hline 669420 & $2014-12-16$ 12:05:00 & & $\begin{array}{l}41,21,04,29,42,05,39,43,13,24 \\
06,79,22,37,12,68,25,36,08,67\end{array}$ \\
\hline
\end{tabular}

Fig 5: Web interface of the lottery

\section{CONCLUSION}

This design aims to solve the problems of analysis data for lottery fans and the difficulty for the real-time web data acquisition. Using LabVIEW to design a web page data realtime acquisition and analysis software, the software realizes the function of real-time update, data acquisition, analysis, displaying and software error alarming, etc. After preliminary test, the software basically completed the data collection, processing, analysis, alarm, display and update functions. Its innovation lies in the supervision of industry, web data collection method, data statistics and analysis of preliminary. To some extent it brings convenience for lottery fans, and it has a certain reference value and practical value. What is more, this method can be effectively applied to the Internet so it display even. So it is with the same size and value, on the single and double, odd-even plate, lower plate, the plate number verification. Therefore, it can be confirmed that the software meets the design requirements.

web information processing in the future, for the purpose of realizing the function of effective information filtration, separation and extraction to users.

\section{REFERENCES}

[1] Ying Nie, Xiangjun Feng. Research on state Machine Model Based on LabVIEW [J]. Hunan: College of Information Engineering. 2007.

[2] Xiumei Wang. LabVIEW Applications in Analog Electronic Circuit Design and Simulation [J].Nanjing: Department of Information Science. 2013

[3] Guoming Liu. virtual instrument system based on the LabVIEW [J]. Hebei: Ordnance Engineering College. 2009 
[4] Guangpu Liu, Xiaodong Li. Weather forecast system based on LabView[J]. Journal of electronic measurement technology, 2014

[5] YanYan Zengqiang Ma Ming Yang. Based on LabView data acquisition and process software programming skills [J].Microcomputer information, 2005

[6] Peigang Jia. Sirui He, Lan Duan. Multi-channel real-time data processing system based on LabView design [J].Journal of manufacturing automation. 2014

[7] K.D.Kim, Rizwan-uddin. A web-based nuclear simulator using RELAP5 and LabVIEW Nuclear Engineering and Design [J], Volume 237, Issue 11, Pages 1185-1194
[8] Ye Yu, Yangan Zhang. A LabVIEW-based realtime measurement system for polarization detection and calibration [J]. Optik 125(2014) 2256-2260

[9] L. Giannone, T. Eich, J.C. Fuchs, M. Cerna, S.Concezzi. Data acquisition and realtime bolometer tomography using LabVIEW RT [J]. Fusion Engineering and Design 86 (2011) 11291132

[10] Wang Mingang, Li Wei. Application of NI Real-Time Hypervisor in Bellows Swing Measurement and Control System [J]. Procedia Engineering 00 (2011) 000-000 